\title{
Physico-Chemical Changes of Frozen Chicken Burger Formulated with Some Spices and Herbs
}

\author{
Soumia M. I. Darwish ${ }^{1}$, Mohamed A. H. El-Ge ddawy ${ }^{1}$, Re da M. B. Khalifa ${ }^{2}$, Rewaa A. A. Mohame d ${ }^{2, *}$ \\ ${ }^{1}$ Food Science and Technology Dept. Faculty of Agric. Assiut Univ. Assiut, 71526, Egypt \\ ${ }^{2}$ Food Technology Research Institute, Agricultural Research Center, Giza, 12619, Egypt
}

\begin{abstract}
The main objective of the present study was to examine the visibility of using natural spices and herbs (thyme, rosemary, sage, marjoram and black seeds) to help chicken burger industry to improve physical properties and extend the shelf life of chicken burger. Two concentrations of these spices $(0.5$ and $1 \%)$ were employed on chicken burger stored at $18^{\circ} \mathrm{C}$ for 6 months. Study indicated that, there were slight decreases in $\mathrm{pH}$ values, water holding capacity (W.H.C) and cooking yield in all treatments throughout the frozen storage period. Furthermore, during storage the drip loss, cooking loss and shrinkage was progressively increased by the extending of storage in all treatments. Meanwhile, treatments which had spices and herbs at levels $1 \%$ showed slight increase in $\mathrm{pH}$ values, water holding capacity (W.H.C) and cooking yield compared with control and treatments which had spices and herbs at level $0.5 \%$. Also, the results appeared that control sample was recorded the highest percentage of drip loss, cooking loss and shrinkage than other treatments. Furthermore, the treatments containing spices and herbs at level $0.5 \%$ had higher drip loss, cooking loss and shrinkage than those treatments containing spices and herbs at level $1 \%$.
\end{abstract}

Keywords Physical Properties, Chicken Burger, Thy me, Sage, Rosemary, Marjoram, Black Seeds

\section{Introduction}

Poultry meat is widely accepted as a good source of high-quality protein. Chicken had some important physical properties. Water holding capacity and $\mathrm{pH}$ several methods have been applied to determine water holding capacity, such as the bag drip method or the filter paper compression method[1]. Water holding capacity is one of the most important qualitative characteristics of meat, it can affect the appearance of the product, its behavior on cooking and its juicy sensation on chewing[2].

During thawing of frozen meat the separation of drip is one of the problems facing the meat technologists. The drip separation causes loss in weight and in nutritive value since drip contains valuable nitrogenous compounds, minerals, and vitamins and might lead to the sensation of dryness and loss of juiciness[3].

Cooking loss, cooking yield and shrinkage considered the most important quality attributes of meat products for both consumers and meat products producers technologists [4]. The objective of the present study was investigate the feasibility of using selected natural spices and herbs for improving the physical properties and extending the shelf life of chicken burger during freezing storage.

* Corresponding author:

rewaaahmed@ymail.com (Rewaa A. A. Mohamed)

Published online at http://journal.sapub.org/s

Copyright (C) 2012 Scientific \& Academic Publishing. All Rights Reserved

\section{Materials and Methods}

\subsection{Materials}

\subsubsection{Chicken Meat}

$10 \mathrm{~kg}$ of fresh chicken meat from broiler carcasses (7-8 weeks age with an average weight $1.5-2 \mathrm{~kg}$ ) were obtained from El-Borssa Company for Poultry at February 2010. On receipt at the laboratory, they were washed carefully then deboned within two hours of slaughtering, the chicken meat was minced using a meat mincer and then chilled at $4 \pm 1^{\circ} \mathrm{C}$ for 24hours before using in processing of chicken burgers.

\subsubsection{Selection of Spices and Herbs}

Selected spices and herbs were used in chicken burger formula namely thyme (thymus vulgaris L.), rosemary (Rosmarinus officinalis L.), black seeds (Nigella sativa L.), sage (Salvia officinalis), and marjoram (Origanum majoranum), were obtain from the Agricultural Research Center, Giza, Egypt.

\subsubsection{Salt, Onion, Whole Egg and Bread Crust Powder}

Salt, onion, whole egg and bread crust powder were obtained from the local market and used for preparation of chicken burger. While, soy flour was purchased from the Food Technology Reasearch Institute. Agricultural Research Center-Giza, Egypt. 


\subsection{Methods}

\subsubsection{Preparation of Chicken Burger}

Fresh chicken burger samp les were prepared as described by [5]. All ingredients were minced twice, after mincing, the chicken mixture was shaped manually using a patty marker (stainless steel model "Form") to obtain round discs $10 \mathrm{~cm}$ diameter and $0.5 \mathrm{~cm}$ thickness. Burgers were packaged in polyethylene bags (in foam dishes).

- The Basal constituents of chicken burger were prepared as follows:

The chilled minced chicken meat formula included fat $71.5 \%$, fresh onion (finely ground) $7.0 \%$, whole egg (blended) $5.0 \%$, bread crust powder $5.0 \%$, rehydrated extruded soy $10.0 \%$ and sodium chloride $1.50 \%$. These ingredients were mixed together, divided to eleven equal portions, the first portion was remained without any addition (control) and the ten reminder portions were individually mixed with two concentrations of each spices and herbs $(0.5 \%$ and $1 \%)$ to give ten treatments. All burgers treatments and control were freeze stored at $-18 \pm 2^{\circ} \mathrm{C}$ up to 6 months.

\subsubsection{Physical Methods}

Fresh chicken meat used in this study was analyzed immediately upon receipt at the laboratory for physical analyses, as well as immediately after manufacturing (zero-time analyses), and then after 1, 2, 3, 4, 5 and 6 months of fro zen storage at $-18 \pm 2^{\circ} \mathrm{C}$.

\subsubsection{1. $\mathrm{pH}$ Value}

$\mathrm{pH}$ values of fresh meat and treated samples were measured in a homogenate prepared with $10 \mathrm{~g}$ sample and distilled water $(100 \mathrm{ml})$, using ICM $41150 \mathrm{pH}$ meter[6].

\subsubsection{Water Holding Capacity (W.H.C)}

Water holding capacity (WHC) was measured using the method of[7] as follows:

Minced chicken burger sample $0.3 \mathrm{~g}$ was placed on an ashless filter paper Whatman, No. 41 and placed between two glass plates, and pressed for 10 minutes by one $\mathrm{kg}$ weight, two zones were found on the filter paper, their surface areas were measured by a planimeter. The outer zone resulted from the water separated from the pressed tissues thus indicating the water holding capacity.

\subsubsection{Drip Loss}

Drip loss was measured by the difference between weight of complete frozen burger and weight of the same burger after thawing. The drip loss was calculated as the percentage of weight change[8].

\subsubsection{Cooking Loss}

Cooking loss of the prepared chicken burger was determined according to[9]. Cooking loss was calculated after grilling of chicken burger as follows:

$$
\% \text { Cooking loss }=\frac{\text { Raw sample weight }- \text { cooked sample weight }}{\text { Raw sample weight }} \times 100
$$

\subsubsection{Cooking Yield}

Cooking yield was calculated as given by[10].

$\%$ Cooking yield = 100 - \% Cooking loss

\subsubsection{Shrinkage}

The shrinkage percentage was calculated as described by[9] as follows:

$\%$ shrinkage $=$

$\frac{(\text { Raw thickness }- \text { Cooked thickness })+(\text { Raw diameter }- \text { Cooked diameter })}{(\text { Raw thickness }+ \text { Raw diameter })} \times 100 \%$

\subsubsection{Statistical Analys es}

The data obtained from three replicats were analyzed by ANOVA using the SPSS statistical package program, and differences among the means were compared using the Duncan's Multiple Range test[11]. At a significance level of 0.05 was chosen.

\section{Results and Discussion}

\section{1. pH Value}

Measuring of $\mathrm{pH}$ value is an important because of its influence on many characteristics, including shelf-life, color, water holding capacity and texture of meat and meat products [12].

The $\mathrm{pH}$ value of different chicken burger treatments during frozen storage at $-18^{\circ} \mathrm{C}$ up to 6 months were tabulated in Table (1). From these data, it could be noticed that different chicken burger treatments had less $\mathrm{pH}$ value than fresh chicken meat ( $\mathrm{pH}$ 5.80). The decreasing in $\mathrm{pH}$ might be attributed to the ingredient used in the burger formula as some of them have acidic effect. Moreover, addition of spices and herbs increased $\mathrm{pH}$ values of burger formula. These results were in agreement with[13].

During fro zen storage of different treatments at $-18^{\circ} \mathrm{C}$ for 6 months, slight and not detectable decrease of $\mathrm{pH}$ values were observed. In this concern,[14] and[15] found that, the $\mathrm{pH}$ values of sausage decreased during storage time at $-18^{\circ} \mathrm{C}$ increased, and attributed this decrease to the breakdown of glycogen to produce lactic acid.[16] studied the influence of rosemary and sage extracts on deboned chicken meat (DCM) quality during refrigerated and frozen storage, and they found that $\mathrm{pH}$ values of treated and untreated DCM decreased as the storage periods increased.[17] stated that the effect of various levels of rosemary or chinese mahogany on the quality of fresh chicken sausage during refrigerated storage, they observed that smaller $\mathrm{pH}$ reduction was observed for the samples with more rosemary or Chinese mahogany added. The $\mathrm{pH}$ reduction was probably due to the fact that some existing oxygen inside the package might trigger fat oxidation, thus resulting in the decrease of $\mathrm{pH}$ values.

Also, from the same table, it could be noticed the addition 
of both spices and herbs to the basal chicken burger formula led to no significantly increase in $\mathrm{pH}$ values during frozen storage except in samples formulated with rosemary $0.5 \%$, sage $0.5 \%$ and marjo ram $0.5 \%$. Moreover, data showed that the control sample had the lowest value in $\mathrm{pH}$ with a significant difference than other treatments in most cases. Also, the addition of spices and herbs at level $1 \%$ caused significant increase in $\mathrm{pH}$ value compared with the addition of spices and herbs at level $0.5 \%$ in some cases.

\subsection{Water Holding Capacity (W.H.C)}

The water holding capacity W.H.C of meat is defined as the ability of meat to hold fast to its own or added water

during processing. It is considered as an important factor affects eating quality, tenderness, juiciness, thawing drip and cooking loss of meat [18]. This property is largely affected by the muscle protein and the level of $\mathrm{pH}$ value. Moreover, relationship between tenderness and the W.H.C of meat protein was reported by[7] and [19].

Water holding capacity of meat considered as one of the important measurements of quality attributes for determining the possibility of using this meat in manufacturing of meat product. The water holding capacity W.H.C of different chicken burger treatments was determined by filter press method and calculated as percentage of bound water, are shown in Table (2).

The results indicated that although W.H.C of all treatments was convergent at zero time, the control sample (basal burger formula without addition) showed decreased in W.H.C compared with other treatments. Also, treatments which had spices and herbs at level 1\% showed slight increase in W.H.C value compared with control and treatments which had spices and herbs at level $0.5 \%$. In this concern,[15] found that the (W.H.C) of the cooked sausage samples increased as the concentration of cardamom volatile oil and added emulsifiers increased. These results agree with the results obtained with shrinkage data.

Generally, with the progression of storage periods, the W.H.C had declined continuously with a significant statistical difference. The loss of W.H.C by freezing storage might be attributed to protein denaturation and losses in protein solubility. These findings were on line with those obtained by[20],[21],[13] and[22].

Moreover, data in Table (2) showed that the control sample showed significant decrease in W.H.C when compared with other treatments in all cases. Also, treatments of chicken burger contained spices and herbs at level $1 \%$ showed significant increase in W.H.C compared with samples contained spices and herbs at level $0.5 \%$ in most of cases. Furthermore, it could be noticed from Table (2) that, at the end of frozen storage periods no considerable changes were noticed between samples. It could be concluded that, W.H.C was not considerably affected by spice.

\subsection{Drip Loss Percentages}

Results in Table (3) cleared that the percentages of drip loss during freezing storage at $-18^{\circ} \mathrm{C}$ up to 6 months of different chicken burger treatments.

Data in Table (3) shown that, the percentage ofdrip loss of all different chicken burger treatments progressively increased with a significant statistical difference by extending storage time, this might be parallel to the development of denaturation and aggregation of protein. These results were in agreement with[23] and[13]. Also, the increase of drip loss percentage after storage periods of all samples might be attributed to the thaw rigor that might occurred if the meat which was frozen before the adenosine triphosphate ( ATP) has been fallen appreciably, this also might led to the increase of shrinkage at the first periods of storage. Similar results were obtained by[24].

Moreover, it could be noticed that the control sample was recorded the highest significant percentage of drip loss than other treatments in most cases except samples formulated with rosemary at levels 0.5 and $1 \%$ after first month. Also, the treatments formulated with spices and herbs at level $1 \%$ had lower significant percentages of drip loss compared with treatments formulated with spices and herbs at level $0.5 \%$ in some cases.

\subsection{Cooking Loss Percentages}

Cooking loss was measured by the difference between weight of frozen chicken burger and weight of the same chicken burger after cooking[8].

The percentages of cooking loss of different chicken burger treatments, during frozen storage at $-18^{\circ} \mathrm{C}$ up for 6 months are tabulated in Table (4).

The cooking loss of all samples progressively significant increase as the period of storage increased. The highest increase observed after the first month of storage, the rate of increase in cooking loss after one month of storage was low and increased slowly till the sixth month of frozen storage at $-18^{\circ} \mathrm{C}$. These results were parallel to the drip loss due to the fluids separated by cooking might included the fluids which probably separate by the thawing of the fro zen burger.

The progressive increases which were shown by extending storage time might be due to protein denaturation leading to deterioration of water holding capacity (W.H.C). These results are in agreement with[25] and[13]. Consequently, cooking loss results were on line with the changes in (W.H.C), the highest cooking loss corresponded with the lowest (W.H.C). Th is conclusion was previously emphasized by[21] who found that the cooking loss of sausage increased as the period of storage increased up to 90 days at $-18^{\circ} \mathrm{C}$. Furthermore,[26] reported that increasing of cooking loss during freezing storage is probably due to excessive fat separation and water released which occurred during cooking.

Moreover, the highest significant cooking loss was recorded for chicken burger formula (as a control), when compared with other treatments in all cases. As well as, treatments containing spices and herbs at level $0.5 \%$ had significant higher cooking loss than those of treatments containing spices and herbs at level $1 \%$ in all of cases. 


\begin{tabular}{|c|c|c|c|c|c|c|c|c|c|}
\hline $\mid \begin{array}{l}z \\
\mathbb{Z} \\
\mathscr{q}\end{array}$ & 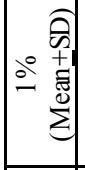 & $\begin{array}{l}n \\
\hat{+} \\
\text { मै } \\
\hat{n} \\
\text { nे }\end{array}$ & $\begin{array}{l}\hat{n} \\
0 \\
0+0 \\
\tilde{D} \\
\sim\end{array}$ & $\begin{array}{l}\text { N } \\
\text { Ò } \\
\text { of } \\
\text { in } \\
\text { nn }\end{array}$ & 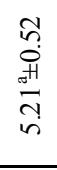 & 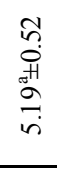 & 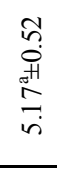 & 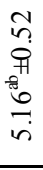 & $\tilde{z}^{2}$ \\
\hline $\mid \begin{array}{l}\frac{\pi}{0} \\
\frac{\pi}{m}\end{array}$ & 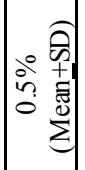 & 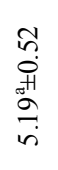 & 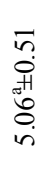 & $\begin{array}{l}n \\
0 \\
0 \\
0 \\
0 \\
i n \\
i n\end{array}$ & 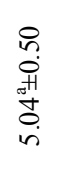 & $\begin{array}{l}0 \\
0 \\
0 \\
\text { of } \\
0 \\
0 \\
\dot{n}\end{array}$ & $\begin{array}{l}n \\
0 \\
0+1 \\
0 \\
0 \\
\dot{0}\end{array}$ & 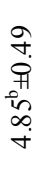 & $\bar{z}$ \\
\hline \multirow{2}{*}{ 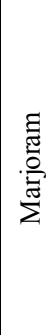 } & 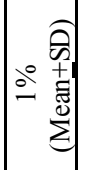 & 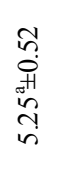 & $\begin{array}{l}\tilde{N} \\
\hat{0} \\
\text { on } \\
\hat{n} \\
\text { nn }\end{array}$ & $\begin{array}{l}\tilde{n} \\
0 \\
0 \\
0 \\
\text { yn }\end{array}$ & 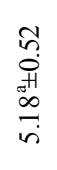 & 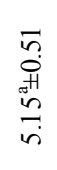 & 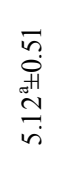 & $\begin{array}{l}\bar{n} \\
\text { †े } \\
\text { की } \\
\infty \\
\text { in }\end{array}$ & $\tilde{z}$ \\
\hline & 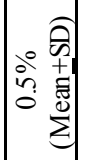 & 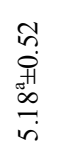 & $\begin{array}{l}0 \\
0 \\
0 \\
\text { d } \\
\text { in }\end{array}$ & 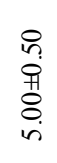 & $\begin{array}{l}\text { oे } \\
\text { Oे } \\
\text { +े } \\
\dot{+}\end{array}$ & 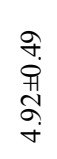 & 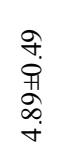 & 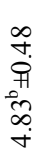 & $*$ \\
\hline \multirow{2}{*}{$\begin{array}{l}80 \\
\text { s. } \\
\text { שn }\end{array}$} & 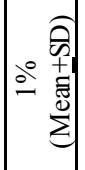 & 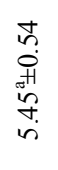 & 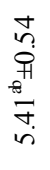 & 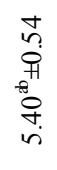 & $\begin{array}{l}+ \\
n \\
o+1 \\
\text { के } \\
\text { nे } \\
\text { nे }\end{array}$ & $\begin{array}{l}+ \\
n \\
0 \\
\text { की } \\
\text { हैं } \\
n \\
n \\
n\end{array}$ & $\begin{array}{l}n \\
n \\
0 \\
\text { †ा } \\
\tilde{n} \\
n \\
n\end{array}$ & $\begin{array}{c}n \\
n \\
0 \\
\text { on } \\
0 \\
0 \\
n \\
n\end{array}$ & Z \\
\hline & $\begin{array}{l}0 \\
\text { a } \\
+1 \\
=\end{array}$ & 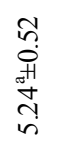 & 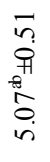 & 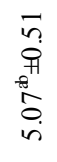 & 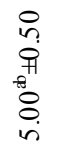 & 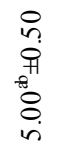 & 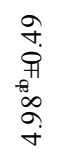 & $\begin{array}{l}\stackrel{a}{+} \\
\text { tे } \\
\stackrel{+}{0} \\
\dot{+}\end{array}$ & $*$ \\
\hline \multirow{2}{*}{ 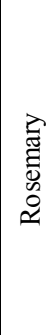 } & 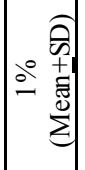 & $\begin{array}{l}n \\
n \\
o+ \\
\pm n \\
n \\
n \\
n\end{array}$ & 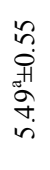 & $\begin{array}{l}n \\
n \\
0 \\
0+1 \\
50 \\
\vdots \\
\dot{n}\end{array}$ & 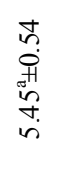 & 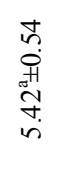 & 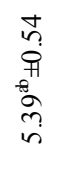 & 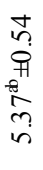 & Z \\
\hline & 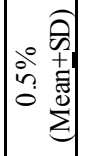 & 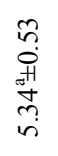 & $\frac{n}{n}$ & $\begin{array}{l}n \\
\hat{n} \\
0+1 \\
0 \\
0 \\
n \\
n\end{array}$ & $\begin{array}{l}n \\
\hat{i} \\
\text { Ð } \\
\hat{n} \\
\hat{n}\end{array}$ & 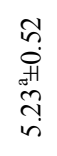 & $\begin{array}{l}\infty \\
\stackrel{0}{+} \\
\stackrel{+}{+} \\
\stackrel{0}{0} \\
\stackrel{+}{+}\end{array}$ & $\begin{array}{l}\stackrel{r}{+} \\
\text { f. } \\
\stackrel{+}{+} \\
\stackrel{+}{+}\end{array}$ & $*$ \\
\hline \multirow{2}{*}{ 莺 } & 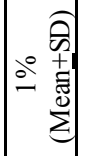 & $\begin{array}{l}0 \\
n \\
o+1 \\
\infty \\
\infty \\
n \\
n\end{array}$ & $\begin{array}{l}0 \\
n \\
0 \\
00 \\
0 \\
n \\
n\end{array}$ & $\begin{array}{l}n \\
n \\
0 \\
\text { in } \\
\text { in } \\
n \\
n\end{array}$ & 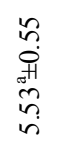 & $\begin{array}{l}n \\
\text { in } \\
0+1 \\
\text { ơ } \\
i n \\
n\end{array}$ & 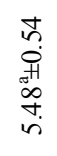 & 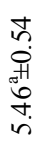 & $\tilde{z}$ \\
\hline & 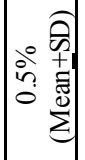 & 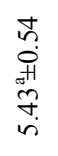 & 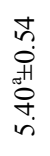 & 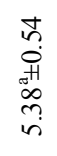 & 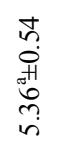 & 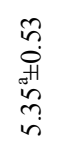 & $\begin{array}{l}n \\
\tilde{n} \\
\text { जी } \\
\tilde{n} \\
\tilde{n}\end{array}$ & $\begin{array}{l}n \\
\text { on } \\
\text { of } \\
\text { on } \\
0 \\
n\end{array}$ & z \\
\hline \multicolumn{2}{|c|}{ 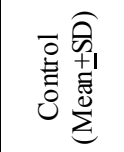 } & 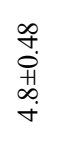 & 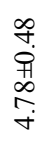 & 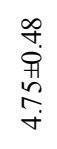 & \begin{tabular}{l}
\multirow{f}{f}{} \\
$\stackrel{+}{0}$ \\
$\stackrel{0}{+}$
\end{tabular} & 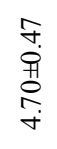 & $\begin{array}{l}\hat{f} \\
0 \\
\text { I } \\
0 \\
0 \\
+\end{array}$ & 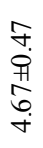 & $\Sigma_{z}$ \\
\hline \multicolumn{2}{|c|}{ 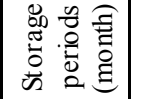 } & 0 & - & $N$ & $m$ & + & in & 0 & $\frac{\infty}{\pi / 2}$ \\
\hline
\end{tabular}

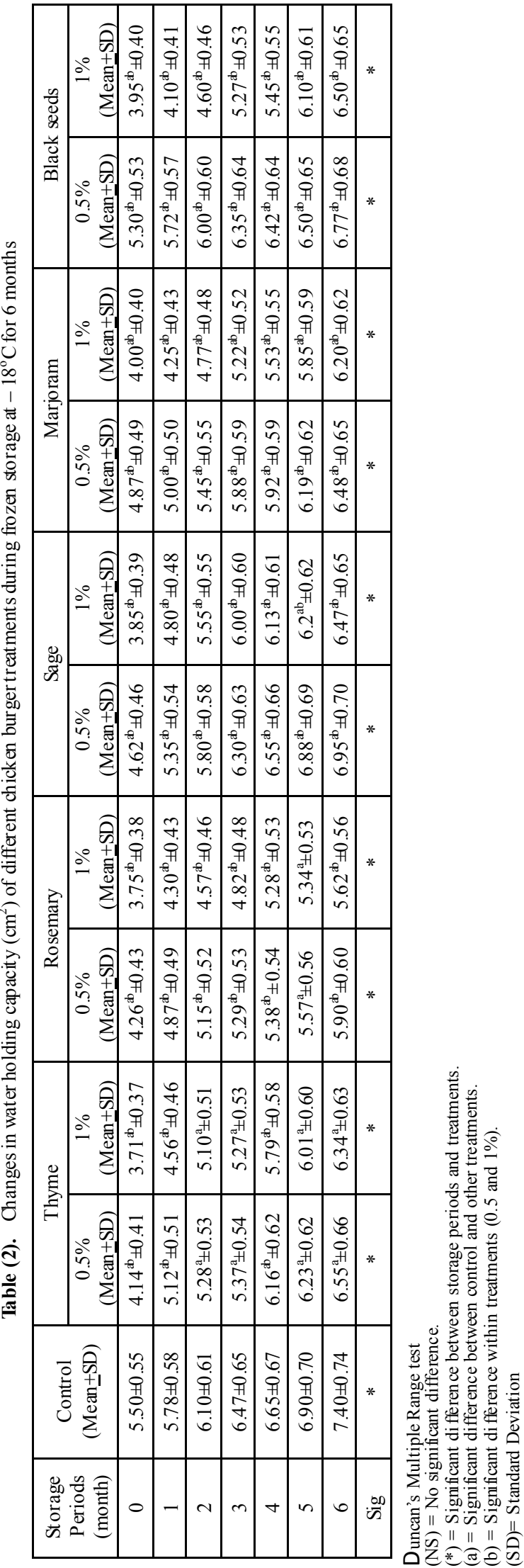




\begin{tabular}{|c|c|c|c|c|c|c|c|c|c|c|}
\hline \multirow{2}{*}{$\begin{array}{l}\frac{n}{0} \\
\mathscr{y} \\
\frac{u}{0} \\
\frac{\pi}{\infty}\end{array}$} & 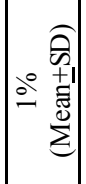 & 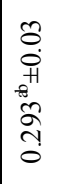 & 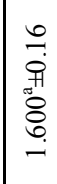 & 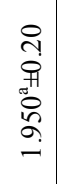 & 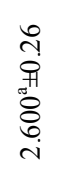 & 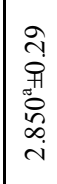 & 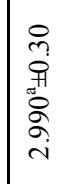 & 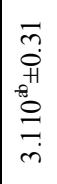 & $*$ & \\
\hline & 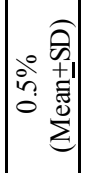 & 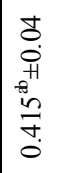 & 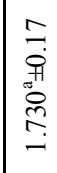 & 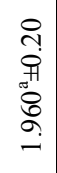 & 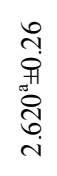 & $\mid \begin{array}{l}0 \\
0 \\
0 \\
0 \\
0 \\
0 \\
0 \\
0 \\
i\end{array}$ & 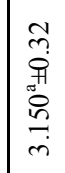 & 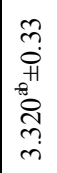 & $*$ & \\
\hline \multirow{2}{*}{ 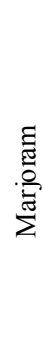 } & 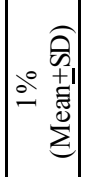 & $\mid \begin{array}{l}n \\
0 \\
0 \\
0 \\
0 \\
0 \\
0 \\
0 \\
0\end{array}$ & $\begin{array}{l}= \\
0 \\
0 \\
0 \\
0 \\
0 \\
0 \\
0 \\
-1\end{array}$ & 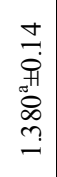 & $\begin{array}{l}0 \\
1 \\
01 \\
0 \\
0 \\
n \\
0 \\
-1\end{array}$ & 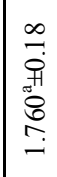 & 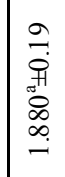 & 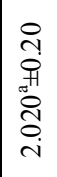 & $*$ & \\
\hline & 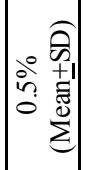 & 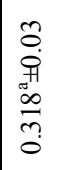 & 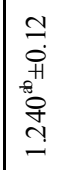 & 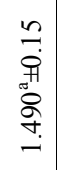 & 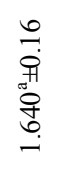 & $\begin{array}{l}0 \\
0 \\
0 \\
0 \\
0 \\
0 \\
0 \\
\infty \\
-1\end{array}$ & 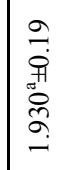 & 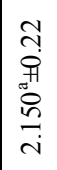 & $*$ & \\
\hline \multirow{2}{*}{ 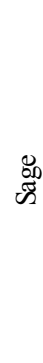 } & 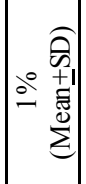 & 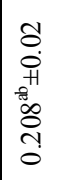 & 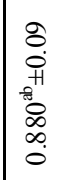 & 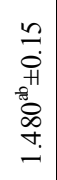 & $\begin{array}{l}0 \\
0 \\
0 \\
+1 \\
0 \\
0 \\
n \\
n \\
-\end{array}$ & $\begin{array}{r}1 \\
0 \\
0 \\
+1 \\
-0 \\
0 \\
0 \\
0 \\
-1\end{array}$ & 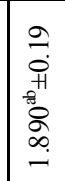 & 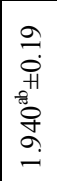 & * & 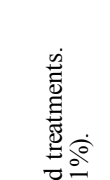 \\
\hline & 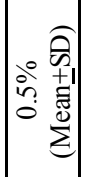 & 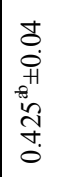 & 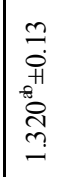 & 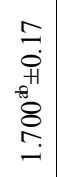 & 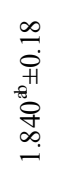 & 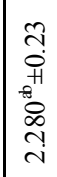 & 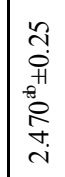 & 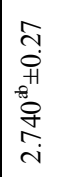 & $*$ & $\begin{array}{l}0 \\
0 \\
0\end{array}$ \\
\hline \multirow[t]{2}{*}{ 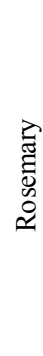 } & 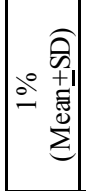 & $\begin{array}{c}0 \\
0 \\
0 \\
+1 \\
0 \\
\infty \\
0 \\
0 \\
0\end{array}$ & 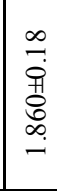 & 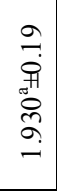 & 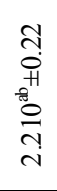 & 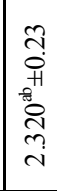 & 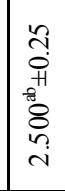 & 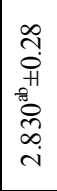 & * & 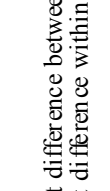 \\
\hline & 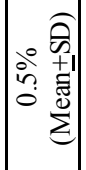 & $\mid \begin{array}{c}0 \\
0 \\
0 \\
0 \\
0 \\
0 \\
0 \\
0 \\
0 \\
0\end{array}$ & 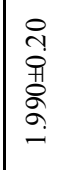 & 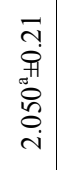 & 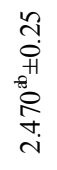 & 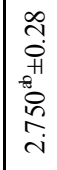 & 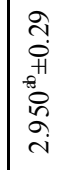 & 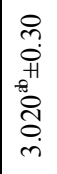 & $*$ & \|\| \\
\hline \multirow{2}{*}{ 胥 } & 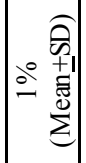 & $\begin{array}{l}0 \\
0 \\
0 \\
0 \\
\tilde{0} \\
0 \\
0 \\
0 \\
0\end{array}$ & 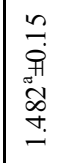 & 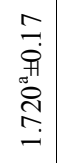 & $\begin{array}{l}\infty \\
\stackrel{\infty}{0} \\
\text { †े } \\
\text { के } \\
\infty \\
\infty \\
-1\end{array}$ & 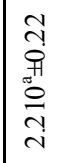 & 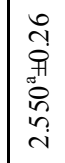 & 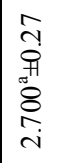 & $*$ & \\
\hline & 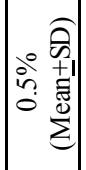 & $\begin{array}{l}t \\
0 \\
0 \\
0 \\
0 \\
0 \\
0 \\
0 \\
0 \\
0\end{array}$ & 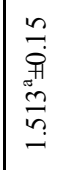 & 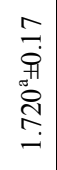 & $\begin{array}{l}\stackrel{9}{0} \\
\stackrel{0}{0} \\
\stackrel{0}{0} \\
\stackrel{\infty}{\infty} \\
-1\end{array}$ & 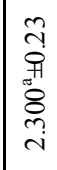 & 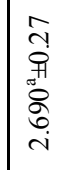 & 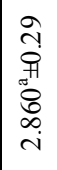 & * & \\
\hline \multicolumn{2}{|c|}{ 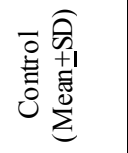 } & $\begin{array}{l}\infty \\
0 \\
0 \\
0 \\
0 \\
0 \\
\infty \\
0 \\
0\end{array}$ & 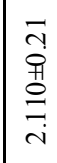 & 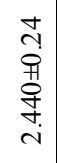 & 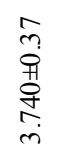 & 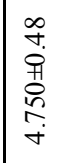 & $\begin{array}{l}\infty \\
0 \\
0 \\
0 \\
0 \\
0 \\
i \\
i\end{array}$ & $\begin{array}{l}\infty \\
0 \\
0 \\
0 \\
0 \\
0 \\
0 \\
0 \\
0\end{array}$ & $*$ & \\
\hline \multicolumn{2}{|c|}{ 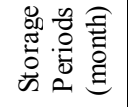 } & 0 & - & $\sim$ & $m$ & $\nabla$ & in & 6 & 100 & \\
\hline
\end{tabular}

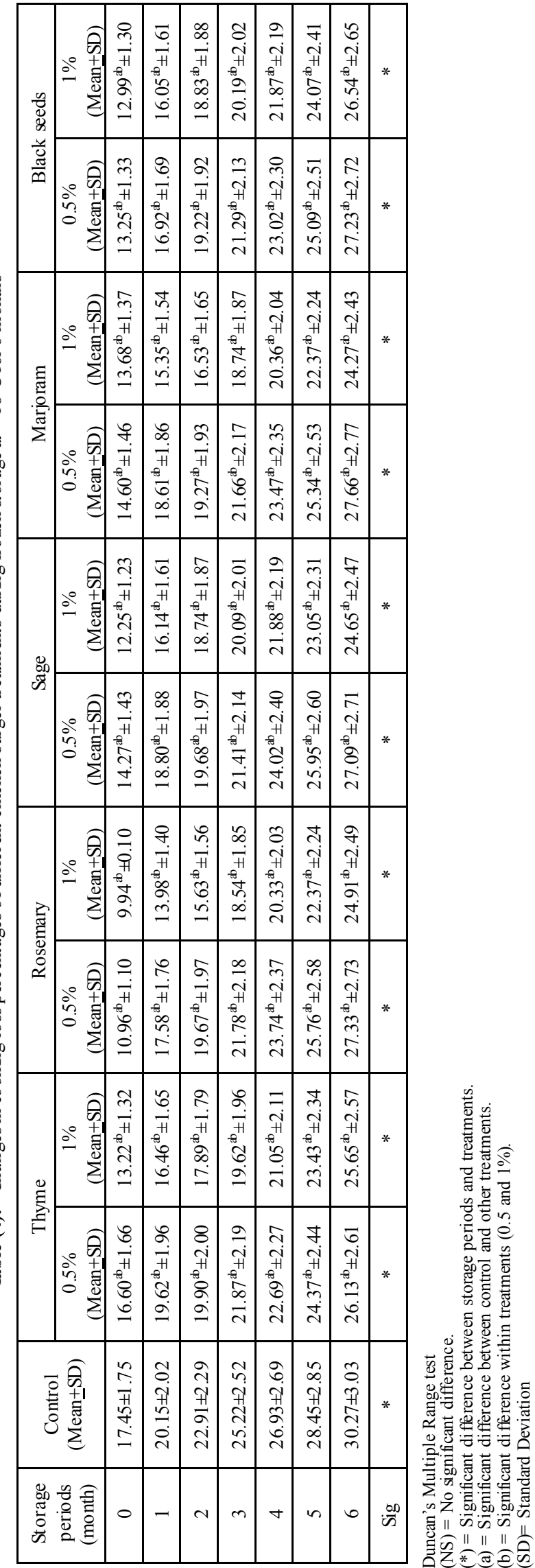




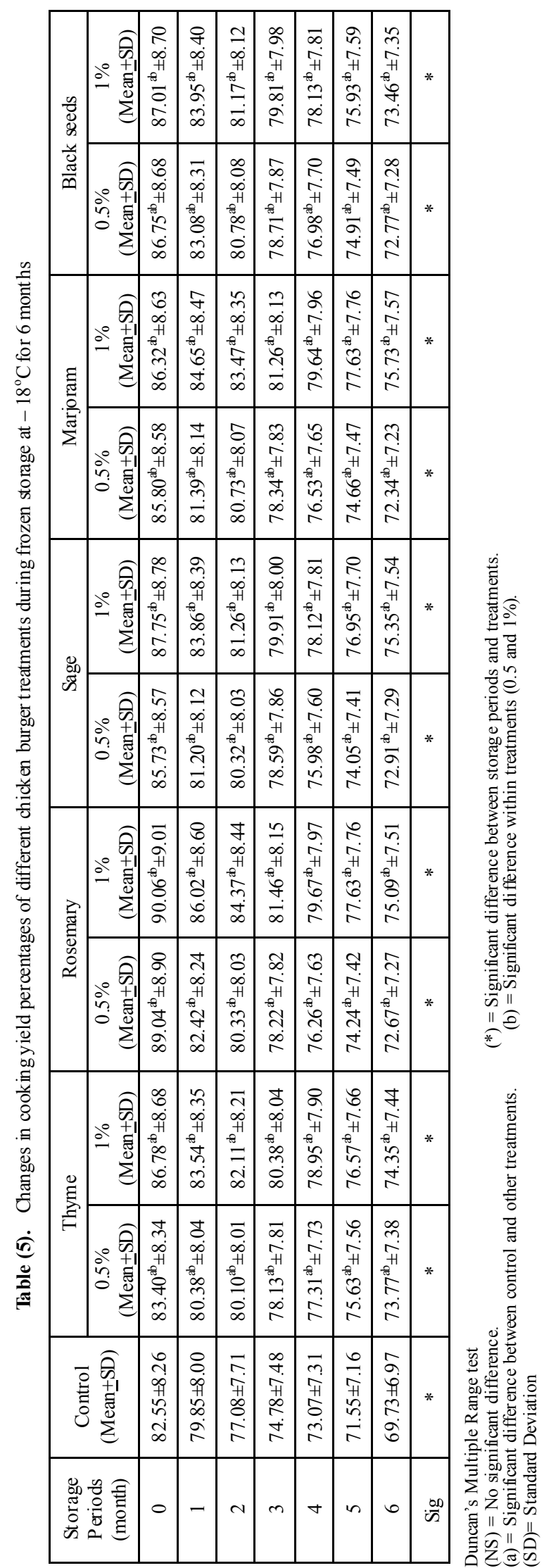

\begin{tabular}{|c|c|c|c|c|c|c|c|c|c|c|}
\hline \multirow{2}{*}{$\begin{array}{l}\tilde{z} \\
\ddot{\Xi} \\
\ddot{y} \\
\ddot{0} \\
\frac{\pi}{m}\end{array}$} & 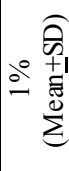 & 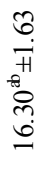 & 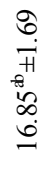 & 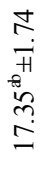 & 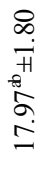 & 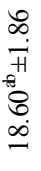 & $\begin{array}{l}\stackrel{8}{8} \\
\text { मे } \\
\stackrel{8}{8} \\
9\end{array}$ & 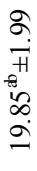 & $*$ & \\
\hline & 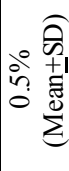 & 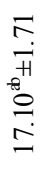 & 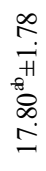 & 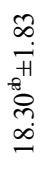 & 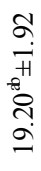 & 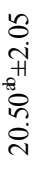 & 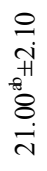 & $\begin{array}{l}\underset{\infty}{\sim} \\
\text { in } \\
\text { d } \\
\stackrel{\infty}{\infty} \\
\stackrel{\sim}{\sim}\end{array}$ & $*$ & \\
\hline \multirow{2}{*}{ 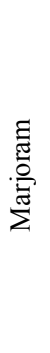 } & 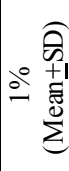 & 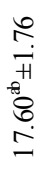 & $\begin{array}{l}n \\
\infty \\
+ \\
+1 \\
0 \\
0 \\
\infty \\
\infty \\
\infty\end{array}$ & $\begin{array}{l}8 \\
\frac{1}{+1} \\
8 \\
8 \\
9 \\
9\end{array}$ & 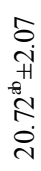 & 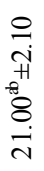 & $\begin{array}{l}\text { I } \\
\text { in } \\
\text { E⿱ } \\
\stackrel{N}{N} \\
\text { i }\end{array}$ & $\begin{array}{l}\text { ते } \\
\text { ते } \\
\text { +1 } \\
8 \\
\text { ते } \\
\text { ते }\end{array}$ & $*$ & \\
\hline & 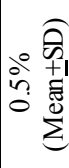 & 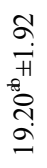 & $\begin{array}{l}\overrightarrow{0} \\
\text { i } \\
\text { + } \\
\text { o } \\
0 \\
\dot{0} \\
\dot{i}\end{array}$ & $\begin{array}{l}8 \\
\text { iे } \\
+ \\
+ \\
\infty \\
\infty \\
\dot{i} \\
0\end{array}$ & 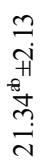 & 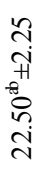 & 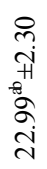 & $\begin{array}{l}\text { त̂. } \\
\text { ते } \\
\text { है } \\
\text { ले } \\
\text { ते } \\
\text { ते }\end{array}$ & $*$ & \\
\hline \multirow{2}{*}{ 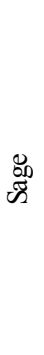 } & 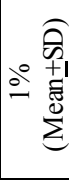 & 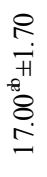 & 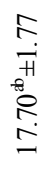 & $\begin{array}{l}\infty \\
\infty \\
+H \\
+1 \\
\infty \\
\infty \\
\infty \\
\infty\end{array}$ & $\begin{array}{l}8 \\
\text { +म } \\
+8 \\
8 \\
9\end{array}$ & 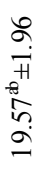 & 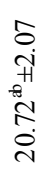 & 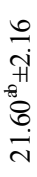 & $*$ & 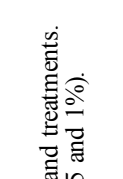 \\
\hline & 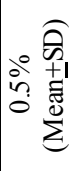 & $\begin{array}{l}8 \\
\text { मे } \\
\text { + } \\
8 \\
8 \\
9\end{array}$ & 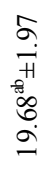 & 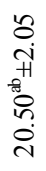 & 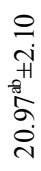 & 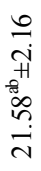 & 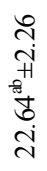 & $\begin{array}{l}\text { ले } \\
\text { ते } \\
\text { के } \\
\text { ते } \\
\text { ले }\end{array}$ & $*$ & 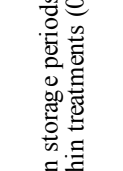 \\
\hline \multirow{2}{*}{ 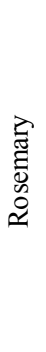 } & 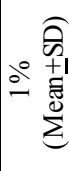 & $\begin{array}{l}8 \\
\infty \\
\dot{H} \\
\text { से } \\
8 \\
\infty \\
\infty\end{array}$ & $\begin{array}{l}\infty \\
\infty \\
+ \\
+ \\
+\infty \\
\infty \\
\infty \\
\infty \\
-1\end{array}$ & 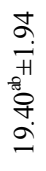 & 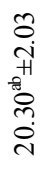 & 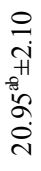 & 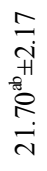 & 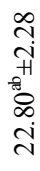 & $*$ & 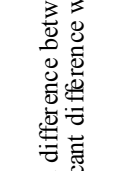 \\
\hline & 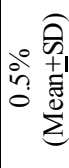 & 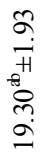 & 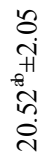 & 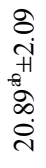 & 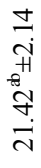 & 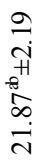 & 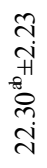 & 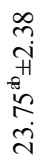 & $*$ & 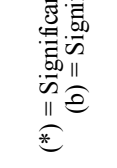 \\
\hline \multirow{2}{*}{ 尽 } & 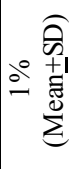 & 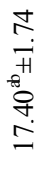 & 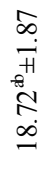 & 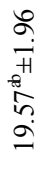 & 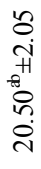 & $\begin{array}{l}8 \\
\dot{0} \\
i \\
+1 \\
\infty \\
\infty \\
\infty \\
\dot{i}\end{array}$ & 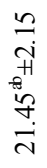 & 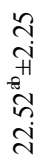 & $*$ & 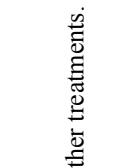 \\
\hline & 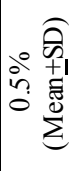 & $\begin{array}{l}\infty \\
\infty \\
+H \\
+\infty \\
\infty \\
\infty \\
\infty\end{array}$ & 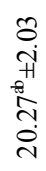 & 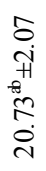 & 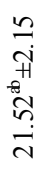 & 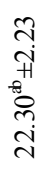 & $\begin{array}{l}8 \\
\text { in } \\
\text { H } \\
+8 \\
8 \\
\ddot{2}\end{array}$ & 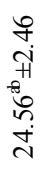 & $*$ & $\overrightarrow{\mathrm{a}}$ \\
\hline \multicolumn{2}{|c|}{ 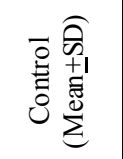 } & 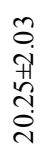 & $\begin{array}{l}\tilde{n} \\
\stackrel{H}{H} \\
\tilde{n} \\
\stackrel{n}{N}\end{array}$ & $\begin{array}{l}\infty \\
\text { त } \\
\text { Tे } \\
\infty \\
\stackrel{i}{i} \\
\text { vi }\end{array}$ & 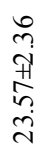 & 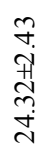 & $\begin{array}{l}0 \\
n \\
\text { ñ } \\
n \\
n \\
n \\
n\end{array}$ & 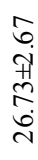 & $*$ & 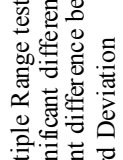 \\
\hline & 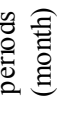 & 0 & - & $\sim$ & $n$ & $\nabla$ & $n$ & 0 & $\ddot{\omega}$ & 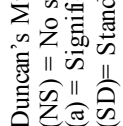 \\
\hline
\end{tabular}




\subsection{Cooking Yield Percentages}

Results in Table (5) cleared that the percentages of cooking yield during frozen storage at $-18^{\circ} \mathrm{C}$ up to 6 months of different chicken burger treatments.

Data represented in Table (5), indicated that with progression of storage periods, the cooking yield had declined continuously of all treatments with a significant difference. In addition, the control sample showed a significant decrease in cooking yield during frozen storage when compared with other treatments in all cases. Furthermore, the treatments containing spices and herbs at level $1 \%$ showed significant increase in cooking y ield when compared with treatments containing spices and herbs at level $0.5 \%$ in all cases.

Generally, the cooking yield occurred in accordance with the changes of cooking loss and (W.H.C) during 6 months storage of chicken burger, the more cooking yield led to the less of cooking loss was found. With advancement of the time of storage, cooking loss progressively increased, while cooking yield decreased. Cooking yield was always higher and cooking loss lower for all treatments. These results were on line with[27].

\subsection{Shrinkage Percentages}

The shrinkage was measured by difference between two dia meters of burger before and after cooking. Moreover, it can be considered as one of important quality attributes measurements. The obtained results were shown in Table (6).

From these results, it could be observed that the control sample showed significant increase in shrinkage compared with other treatments. While, treatments which had spices and herbs at level $0.5 \%$ showed significant higher in shrinkage than treatments which had spices and herbs at level $1 \%$. Also, from the same table, it could be observed that the shrinkage was progressively significant increased by the extending storage time in all treatments. This might be attributed to excessive fat separation and water released which occurred during cooking and decreasing in water holding capacity (W.H.C). These results were on line with the results obtained with cooking loss and drip loss, but they were discrepant with the results of water holding capacity (W.H.C). Similar results were obtained with[13] and[27].

\section{Conclusions}

Results of the present study demonstrate the positive effects of spices and herbs, added with two concentrations to help chicken burger industry to improve physical properties and extend the shelf life of chicken burger during frozen storage at $\left(-18^{\circ} \mathrm{C}\right)$ for 180 days. The best results were obtained with the concentration of spices and herbs at level $1 \%$ to improve physical properties of chicken burger. Treatments which had spices and herbs at levels $1 \%$ showed slight increase in $\mathrm{pH}$ values, water holding capacity (W.H.C) and cooking yield compared with control and treatments which had spices and herbs at level $0.5 \%$. Also, the results appeared that control sample was recorded the highest percentage of drip loss, cooking loss and shrinkage than other treatments. Furthermore, the treatments containing spices and herbs at level $0.5 \%$ had higher drip loss, cooking loss and shrinkage than those treatments containing spices and herbs at level $1 \%$. Physical properties is one of the technological characteristics to judge the quality and appearance of meat and poultry products so the determination is given an indicator to judge the quality of the products

\section{ACKNOWLEDGEMENTS}

The authors thanks Meat and Fish Technology Department Research Institute and Medicinal and Aromatic Plants, Horticulture Research Institute, Agriculture Research center (A.R.C), Ministry of Agriculture, Giza, for support and helps to obtain spices and herbs which used in this investigation.

\section{REFERENCES}

[1] URBIN, M. C., D. A. ZESSIN, B. D. WILSON (1962): Observation on a method of determining the water-binding properties of meat. J. Anim. Sci. 21, 9-13.

[2] WOELFEL, R. L., C. M. OWENS, E. M. HIRSCHLER, R. MARTINEZ-DAWSON, A. R. SAMS (2002): Characterization and the incidence of pale, soft, and exudative broiler meat in a commercial processing plant. Poultry Sci. 81, 579-584.

[3] Garza, A. E. R and Zayas, J. F. (1996). Quality of broiled beef patties supplemented with wheat germ protein flour. J. of food Science, 61 (2): 418-421.

[4] El-Seesy, T. A. (2000). Quality and Safety of meal burger patties using" HACCP System 3. Conference of " The food Industry at the serice of Turisum April 112-14 ${ }^{\text {th }}$ ShrinkageCooking loss- Drip loss.

[5] Turhan, S.; Sagir, I. and Ustun, N. S. (2005). Utilization of hazelnut pellicle in low-fat beef burgers. Meat Sci., 71, 312-316.

[6] Wierbicki, E. \& Deatherage, F.E. (1958). Determination of water- holding capacity of fresh meat. J. Agric. food Chem., 6: 387- 389 .

[7] El-Seesy, T. A. (2000). Quality and Safety of Meal Burger Patties Using “ HACCP” System 3. Conference of " The food Industry at the Service of turisum" April 112-14th Shrinkage-Cooking loss-Drip loss.

[8] A.M.S.A. (1995). Research Guidelines for Cookery, Sensory Evaluation and Instrumental Tenderness Measurements of Fresh Beef. American Meat Science Assoc., Chicago, U.S.A., 240pp. 
[9] El-Nemer, S. E. (1979). Studies on Meat Substitutes. M.Sc Thesis, Faculty of Agric., Zagazig Univ., Zagazig, Egypt.

[10] SPSS. (1998). SPSS for windows. Release, 9.0.0, Standard Version SPSS. Inc.

[11] Clarke, A. D.; Sofos, J. N. and Schmidt, G. R. (1988). Effect of algin / Calcium binder levels on various characteristics of structured beef. J. Food Sci., 53: 711-713.

[12] Abd-El-Qader, M. F. (2003). Quality Improvement of Chicken Frozen Burger Formulated with Some Spices or their Volatile Oils. M. Sc. Thesis, Food Science and Technology Dept., Faculty of Agric., Cairo Univ., Egypt.

[13] El-Shawaf, A. M. (1990). Microbial Studies on Aflatoxins in Meat Products. Ph. D. Thesis, Food Technology Dept., Faculty of Agriculture, Mansoura Univ., Egypt.

[14] El-Harrery, A. S. (1997). Effect of Cardamom Oil on Chemical, Microbiological and Sensory Attributes of Beef Sausage. M.Sc. Thesis, Food Science and Technology Dept., Faculty of Agric., Cairo Univ., Egypt.

[15] Abou Arab, A. A. and Abou Arab, E. A. (2004). Infulence of rosemary and sage extracts on deboned chicken meat quality. Minufiya J. Agric. Res. Vol. 1 No. 29: 169-180.

[16] Liu, D. C.; Tsau, R. T.; Lin, Y.C.; Jan, S. S. and Tan, F. J. (2009). Effect of various levels of rosemary or Chinese mahogany on the quality of fresh chicken sausage during refrigerated storage. Food Chem., 117: 106-113.

[17] Hamm, R. (1986). "Muscle as food". Functional properties of the myofibrillar system and their measurements. pp. 135-199. Elsevier Academic press Inc. Orlando, F.
[18] Morsi, H. H. H. (1988). Studies on Freezing Preservation of Meat. Ph. D. Thesis, Food Science and Technology Dept., Faculty of Agric., Cairo, Univ., Egypt.

[19] Sharaf, S. (1993). Studies on Manufacture of Poultry Sausage. M. Sc. Thesis, Food Science and Technology. Dept. Faculty of Agric., Cairo, Univ., Egypt.

[20] Salama, N. A.; Sharaf, S. M. and Al-Wakil, F. A. (1994). Physical and palatability characteristics of extended chicken sausage. Egypt. J. Food Sci., 22 (2): 293-308.

[21] Hegazy, N. E. (2004). Chemical, Micrbiological and Technological Studies on some Poultry Meat Products. M. Sci. Thesis, Food Industry Dept., Faculty of Agric., El-Mansoura Univ., Egypt.

[22] Abd-El-Baki, M. M.; Taha, R. A.; El-Zayet, F. M. M.; El-Dashlouty, A. A. and Fouda, Z. M. A. (1983). Influence of some pre-freezing treatments on the chemical and physical properties of chicken meat. Proc. 29th Euro. Meeting of meat Res. Workers, Parma, 464.

[23] Lawrie, R. A. (1979). Meat Science. 3rd Ed. Page 300.The eating quality of meat. Pergamon Press. Oxford, England.

[24] Moawad, R. K. (1995). Effect of Pretreatment on Quality Attributes and Nutritive Value of Frozen Beef and Chicken Meats. Ph. D. Thesis, Food Science and Technology Dept., Faculty of Agric.,Cairo Univ. Egypt.

[25] Trius, A.; Sebranek, J. G.; Rust, R. E.; and Carr, J. M. (1994). Low- fat bologna and beaker sausage: Effects of carrageen ans and chloride salt. J. Food Sci., 59 (5): 941-945.

[26] Mohamed, H. A. (2005). Low Fat Products as Prepared from Ostrich and Other Produced Fat Beef. Ph. D. Thesis Nutrition and Food Science. Dept. Faculty of Home Economics. Minufiya University. Egypt 